\title{
INVESTIGACIÓN
}

\section{Aplicación de las técnicas de espacio de cabeza a la extracción de los compuestos orgánicos volátiles de la oleorresina de pimentón}

\author{
Por J. M. Guadayol1, T. Baquero'1 y J. Caixach² \\ 'EUETIT, Dept. Eng. Química. UPC. Colón, 1. 08222 Terrassa (Barcelona). \\ 'Laboratori d'Espectrometría de Masses, CID-CSIC. Jordi Girona, 18-26, 08034 Barcelona, Spain
}

RESUMEN

Aplicación de las técnicas de espacio de cabeza a la extracción de los compuestos orgánicos volátiles de la oleorresina de pimentón.

Se han aplicado las técnicas de espacio de cabeza estático y dinámico para la extracción y concentración de los compuestos orgánicos volátiles de la oleorresina de pimentón. En espacio de cabeza dinámico se han utilizado las variantes de arrastre con borboteo y espacio de cabeza dinámico propiamente dicho. Con el fin de estudiar la influencia de la temperatura en ambas técnicas de extracción, se han realizado pruebas a tres temperaturas diferentes en cada una de ellas, estudiando la influencia de la misma en el proceso de aislamiento de los CVOs. La separación e identificación de los CVOs extraídos se ha llevado a cabo mediante HRGC/MS. Todas las técnicas presentadas son de aplicación simple y reproducibles con facilidad.

PALABRAS-CLAVE: Arrastre por borboteo - Compuestos orgánicos volátiles - Espacio de cabeza dinámico - Espacio de cabeza estático - Oleorresina de pimentón.

\section{SUMMARY}

Aplication of headspace techniques to the extraction of volatile organic compounds from paprika oleoresin

Static and dynamic headspace techniques have been applied to the extraction and concentration of volatile organic compounds (VOCs) from paprika oleoresin. In dynamic headspace, both purge and trap and sweeping techniques have been used. In order to show the influence of temperature on the extraction techniques, three differents temperatures have been applied to each technique. The separation and identification of the extracted VOCs has been carried out through HRGC/MS. The extraction techniques are simple and easily reproducible.

KEY-WORDS: Dynamic headspace - Paprika oleoresin Purge and trap - Static headspace - Volatile organic compounds.

\section{INTRODUCCION}

\subsection{Técnicas de extracción}

Las técnicas de extracción de los componentes volátiles orgánicos (CVOs) de los productos alimenticios son muy variadas y se pueden aplicar varias de ellas, de distinta naturaleza, a un mismo producto. Algunas de estas técnicas requieren temperaturas elevadas que pueden producir cambios químicos en la propia muestra, éste es el caso de la técnica de extracción de Likens y Nickerson (destilación-extracción simultánea, DES) a presión atmosférica en la que el producto se ha de someter a temperaturas superiores a $100^{\circ} \mathrm{C}$ para conseguir la formación de vapor de arrastre.

Para otras técnicas como la extracción mediante disolventes volátiles debe tenerse en cuenta que no se disuelvan otros productos de punto de ebullición elevado, ya que no se podrían someter a una separación por cromatografía de gases. En el caso de la oleorresina de pimentón no tiene sentido extraer los CVOs con un disolvente de naturaleza similar al que se ha utilizado para la obtención industrial de la propia oleorresina (hexano, dicloroetano). La técnica de inyección directa tampoco es recomendable debido a la baja sensibilidad que presenta, Morales et al. (1992).

Las técnicas de espacio de cabeza dinámico fueron introducidas por Swinnerton et al. (1962) y consisten en arrastrar los CVOs de la muestra, sometida a una temperatura determinada, mediante un gas inerte. Los compuestos volátiles son retenidos posteriormente en una trampa adsorbente, que a continuación se somete a desorción mediante un disolvente y se inyecta para su separación por cromatografía de gases. Esta técnica puede desarrollarse de dos modos: haciendo circular el gas superficialmente sobre la muestra sometida a agitación (espacio de cabeza dinámico), o bien introduciendo el gas a través de la muestra también sometida a agitación (arrastre con borboteo). Estas técnicas requieren concentración 
mediante la evaporación parcial del disolvente en el caso que la presencia de volátiles en la muestra sea muy baja.

La técnica de espacio de cabeza estático es de aplicación muy simple, consiste en introducir la muestra en un vial cerrado mediante un septum, aplicar una determinada temperatura y extraer una alícuota de la fracción de vapor con una jeringa para muestras gaseosas, que a continuación se inyectará en el cromatógrafo para la separación de los distintos componentes volátiles.

\subsection{Oleorresina de pimentón}

La elaboración industrial de la oleorresina de pimentón se basa en someter al pimentón (pimiento seco y molido) en forma de pellets a extracción mediante disolventes volátiles, obteniéndose un extracto lipídico en forma de aceite viscoso con un intenso color que contiene todos los componentes del pimentón solubles en el citado disolvente. Para eliminar el disolvente hasta los límites legales permitidos en alimentación, se aplica una destilación con la ayuda de vacío y posteriormente se inyecta vapor de agua para eliminar el disolvente residual; el extracto resultante es la oleorresina de pimentón.

Los componentes volátiles del pimiento de la especie Capsicum annuum $L$. han sido estudiados por diversos autores, Chitwood et al. (1983), Govindajaran (1986) y Wu y Liou (1986). La técnica DES se ha aplicado a la oleorresina de pimentón de tipo Capsicum frutescens de procedencia africana, Keller et al. (1981)

En el presente trabajo la aplicación de técnicas de espacio de cabeza permite trabajar a temperaturas más bajas con la consiguiente disminución de la posibilidad de formación de artefactos.

\section{PARTE EXPERIMENTAL}

Todos los ensayos se han realizado sobre una muestra de oleorresina de pimentón de origen surafricano y procedente de la misma extracción industrial a fin de poder comparar los resultados de las diferentes técnicas aplicadas, de esta forma se evita la comparación entre muestras que hayan sido sometidas a diferentes tratamientos industriales de eliminación de disolvente; no hay que olvidar que la finalidad es la producción de colorante.

\subsection{Espacio de cabeza estático}

Para llevar a cabo esta técnica se ha dispuesto de viales de $43 \mathrm{~mL}$ cerrados herméticamente mediante un septum. Se ha introducido de 1 a $2 \mathrm{~g}$ de muestra en viales y se han llevado a un baño termostatizado, de manera que pueda considerarse que la temperatura del líquido es la de la muestra (una hora). Para realizar la extracción de la fracción de vapor se ha utilizado una jeringa de cromatografía de $1 \mathrm{~mL}$ para muestras gaseosas.

La no existencia de agua en la muestra, teniendo en cuenta el proceso de obtención de la oleorresina de pimentón, facilita mucho los análisis cromatográficos, puesto que el volumen que ocuparía el vapor de agua en el vial viene ocupado por los CVOs presentes en la muestra, lo que aumenta la cantidad de los mismos y la sensibilidad para su detección.

Se han realizado pruebas en un amplio rango de temperaturas, y se han comparado los resultados obtenidos bajo las temperaturas de 60,80 y $100^{\circ} \mathrm{C}$, ya que por debajo de la primera estos han sido muy bajos en rendimientos de CVOs y para temperaturas superiores podrían esperarse efectos térmicos sobre los componentes originales de la oleorresina. Se han realizado tres inyecciones por vial. La extracción de cada fracción volátil produce un desplazamiento del equilibrio de los vapores que se encuentran en el recipiente con lo que se mejora la reproductibilidad, Snyder y Mounts (1990).

\subsection{Espacio de cabeza dinámico}

Se ha hecho circular nitrógeno, en circuito abierto, como gas inerte de arrastre; manteniendo el caudal entre 60 y $90 \mathrm{~mL} / \mathrm{min}$. durante 12 horas, habiendo comprobado que es un tiempo más que suficiente ya que posteriormente el rendimiento no varía entre los límites de caudal citados, resultados coincidentes con los de Núñez et al. (1984).

Se han probado dos materiales adsorbentes para la realización de las primeras pruebas de aplicación de esta técnica en la oleorresina de pimentón: carbón activado y Tenax, aplicando la desorción mediante disolventes en ambos casos.

Las trampas de Tenax se han desorbido con hexano; la poca superficie por unidad de masa del Tenax presenta el problema de una rápida saturación de este adsorbente. El empleo de carbón activado aumenta la eficacia de esta técnica, puesto que su elevada superficie específica la hace más apropiada para este tipo de adsorción. El empleo de trampas de carbón activado se ve favorecido por la ausencia de agua en la oleorresina de pimentón lo que permite aprovechar su mayor superficie específica; en caso contrario el empleo de trampas de Tenax sería posiblemente más adecuado, Núñez et al. (1984). La desorción de las trampas de carbón activado con sulfuro de carbono ofrece la ventaja adicional de que el detector de ionización de llama no presenta una sensibilidad muy elevada a este disolvente. Se han utilizado trampas de carbón de coco activado ORBO-32 (large) con dos compartimientos de 400 y $200 \mathrm{mg}$ de material adsorbente, los resultados negativos obteni- 
dos de la desorción de los 200 mg de cola confirman la no saturación de los primeros $400 \mathrm{mg}$.

Por razones similares a las del apartado anterior se han escogido tres temperaturas representativas, cuyos valores son de 30,50 y $80^{\circ} \mathrm{C}$. Mordret et al. (1985) consideran que trabajando a estas temperaturas los volátiles obtenidos corresponden realmente a los presentes en la muestra y no a volátiles formados por degradación térmica de algunos precursores. Las cargas se consideran suficientes entre 30 y 40 gramos de oleorresina de pimentón.

Lo indicado anteriormente es válido tanto para la técnica de arrastre con borboteo como para la técnica de espacio de cabeza dinámico propiamente dicho.

La figura 1 muestra la disposición del sistema de extracción de arrastre con borboteo. El comportamiento físico de la oleorresina de pimentón favorece el arrastre de CVOs cuando la temperatura aumenta, debido a una disminución de la viscosidad. La mayor fluidez de la oleorresina favorece la acción del nitrógeno en el seno de la misma, permitiendo que se difunda con mayor facilidad; asimismo, hace que la agitación sea más homogénea. La formación de espuma se ha evitado con la utilización de matraces de forma esférica.

La disposición del sistema de extracción mediante espacio de cabeza dinámico es similar a la de la figu- ra 1 con el tubo del gas de arrastre por encima de la masa de oleorresina de pimentón.

\subsection{Análisis cromatográfico, identificación y cuantificación}

El análisis cromatográfico y la cuantificación se han realizado en un equipo Fisons Serie GC8000 con un detector de ionización de llama (FID). Gas portador: helio (100kPa), caudal de $1 \mathrm{~mL} / \mathrm{min}$. Inyección split 1:16. Columna DB-5 (J \& W), $60 \mathrm{~m} \times 0,251 \mathrm{~mm}$, espesor fase estacionaria: $0,25 \mu \mathrm{m}$.

Programa de temperaturas: $50^{\circ} \mathrm{C}$ (isoterma durante 10 minutos) hasta $230^{\circ} \mathrm{C}\left(2^{\circ} \mathrm{C} / \mathrm{min}\right)$. Temperatura del inyector: $250^{\circ} \mathrm{C}$. Temperatura del detector: $250^{\circ} \mathrm{C}$. Inyección: 1-2 $\mu \mathrm{L}$ para las muestras líquidas (espacio de cabeza dinámico) y $1 \mathrm{~mL}$ para las muestras gaseosas (espacio de cabeza estático).

La identificación de los picos se ha realizado mediante HRGC/MS en un equipo MD800 de Fisons con características cromatográficas similares a las anteriores. Los espectros se han obtenido mediante ionización $\mathrm{El}^{+}$a $70 \mathrm{eV}$ con $\mathrm{m} / \mathrm{z}$ entre 45 y 450 a 1s/scan e identificándolos por comparación con las

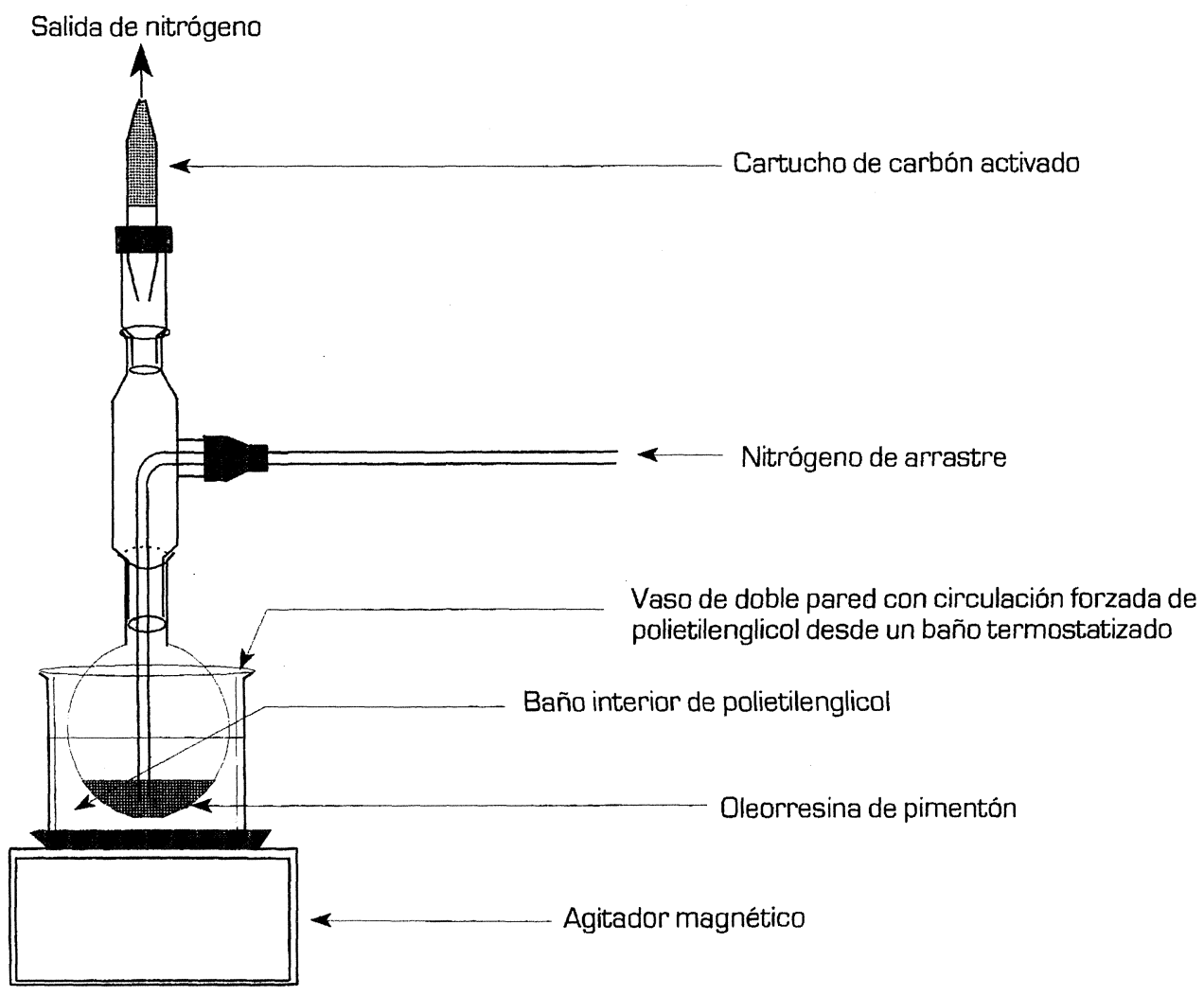

Figura 1

Sistema de extracción de arrastre por borboteo 
bases de datos NIST/NBS y Wiley. Algunos compuestos volátiles se han confirmado además con patrones de referencia.

La cuantificación se ha llevado a cabo mediante la adición de nerol como patrón interno a una muestra de 30,0 gramos, por lo que la concentración de cada componente está referida a éste.

\section{RESULTADOS Y DISCUSIÓN}

\subsection{Técnicas y condiciones de extracción}

La figura 2 muestra un cromatograma de los CVOs obtenidos mediante espacio de cabeza estático obtenido a $80^{\circ} \mathrm{C}$. Se observa que únicamente son detectables aquellos compuestos volátiles que presentan tiempos de retención menores; ello supone que alguno de los componentes que aportan las características más aromáticas, por su baja presión de vapor, se pueden detectar con facilidad mediante este sistema de extracción, que resulta ser sencillo y de rápida aplicación. El rendimiento cualitativo y cuantitativo de la extracción mejora a medida que aumenta la temperatura.

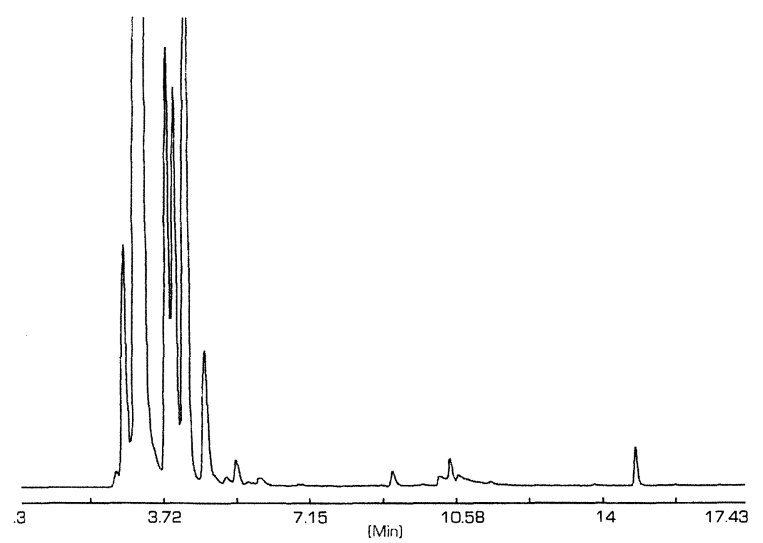

Figura 2

Cromatograma correspondiente a los CVOs obtenidos con el método de espacio de cabeza estático a $80^{\circ} \mathrm{C}$

Comparados los cromatogramas obtenidos de las dos técnicas de espacio de cabeza dinámico se observan un rendimiento cualitativo y cuantitativo superior en la técnica de arrastre con borboteo. Esto se explica porque la circulación del nitrógeno a través de la oleorresina facilita el arrastre de mayor cantidad de CVOs.

Los mejores resultados se han obtenido a la temperatura de ensayo más elevada $\left(80^{\circ} \mathrm{C}\right)$, cuya explicación se encuentra en la disminución de la viscosidad de la oleorresina al aumentar la temperatura lo que facilita la circulación del nitrógeno, a este efecto se añade el aumento de la presión de vapor de los CVOs con la temperatura.

\subsection{Identificación y cuantificación}

La tabla I presenta los compuestos identificados en la oleorresina de pimentón de procedencia surafricana y su concentración en la misma. Es conveniente indicar que las condiciones de extracción industrial de oleorresina puede variar en gran medida la cuantificación de los CVOs.

Tabla I

Cuantificación (con nerol como patrón interno) de los compuestos identificados en la oleorresina de pimentón objeto de estudio

\begin{tabular}{|c|c|}
\hline Compuesto identificado & Concentración (mg/Kg) \\
\hline 1,3-dimetilbenceno & 23,8 \\
\hline 6-metil-5-hepten-2-ona & 0,6 \\
\hline 2,2,6-trimetilciclohexanona & 0,3 \\
\hline tetrametilpiracina & 0,5 \\
\hline 6-metil-3,5-heptadien-2-ona & 1,6 \\
\hline 2,6-dimetilciclohexanol & 1,1 \\
\hline 2,4-dimetilbenzaldehído & 0,2 \\
\hline$\beta$-ciclocitral & 0,5 \\
\hline$\beta$-elemeno & 7,9 \\
\hline Geranilacetona & 2,0 \\
\hline$\beta$-ionona & 1,1 \\
\hline dihidroactinidiolide & 1,4 \\
\hline
\end{tabular}

La figura 3 presenta el cromatograma de los CVOs extraídos mediante la técnica de arrastre con borboteo. La identificación de los compuestos se corresponde con la numeración indicada en el cromatograma.

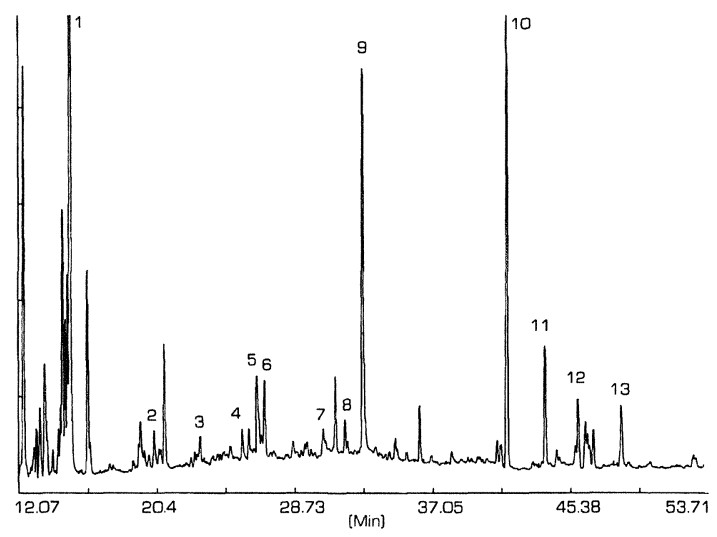

Figura 3

Cromatograma correspondiente a los CVOs identificados a partir de la extracción mediante arrastre por borboteo. 1:1,3dimetilbenceno. 2:6-metil-5-hepten-2-ona. 3:2,2,6-trimetilciclohexanona. 4:tetrametilpirazina. 5:6-metil-3,5-heptadien-2-ona. 6:2,6-dimetilciclohexanol. 7:2,4-dimetilbenzaldehído. 8: $\beta$-ciclocitral. 9: nerol (patrón interno). 10: $\beta$-elemeno. 11:6,10-dimetil5,9-undecadien-2-ona. 12: $\beta$-ionona. 13:dihidroactinidiolide. 


\section{BIBLIOGRAFIA}

Chitwood, R. L., Pangborn R. M. y Jennings, W. (1983) - GGC/MS and Sensory Analysis of Volatiles from Three Cultivars of Capsicum».- Food. Chem. 11, 201-216.

Govindajaran, V. S. (1986). - "Capsicum. Production, Technology, Chemistry and Quality. Part III: Chemistry of the Color, Aroma and Pungency Stimuli, CRC Crit».- Rev. Food Sci. Nutr. 24, 245-355.

Keller, U., Flath, R. A., Mon, T. R. y Teranishi, R. (1981) - "Volatiles from Red Pepper (Capsicum spp.)".Teranishi, R. y Barrera, H., Eds.; Quality of Selected Fruits and Vegetables of North America, ACS Symposium Series 170; American Chemical Society; Washington, DC, pp. 137-146.

Morales, M. T., Aparicio, R. y Gutiérrez, F. (1992). - «Técnicas de aislamiento y concentración de volátiles de aceites vegetales".- Grasas y Aceites 43, 164-173.

Mordret, F., Morin, O. y Coustille, J. L. (1985) - Détermination des flaveurs de corps gras».- Rev. Fr. Corps Gras 32, 193-200.

Núñez, A. J., González, L. F. y Janák, J. (1984). -«Pre-concentration of Headspace for Trace Organic Analysis by Gas Chromatography».- J. Chromatographic Science 300, 127-162.

Snyder, J. M. y Mounts, T. L. (1990). - «Analysis of vegetable oil volatiles by multiple headspace extraction».- J. Am. Oil Chemists'Soc. 67, 800-803.

Swinnerton, J. W., Linnenbom, V. J. y Cheek, C. H. (1962). - «Determination of dissolved gases in aquous solutions by gas chromatography".- Ana. Chem. 34, 483-485.

Wu, C. M. y Liou S. E. (1986). - «Effect of Tissue Disruption on Volatile Constituents of Bell Peppers".- J. Agric. Food Chem. 34, 770-772.

Recibido: Enero 1996

Aceptado: Enero 1997 